Revista de Indias, 1996, vol. LVI, núm. 207.

\title{
JERÓNIMO MÜNZER Y EL DESCUBRIMIENTO DE AMERICA
}

POR

FRANCISCO CALERO

UNED, Madrid

Jerónimo Münzer realizó en los años 1494 y 1495 un viaje por la Península Ibérica, del que escribió un interesante relato. Su relación con América descansa en varios hechos: el 14 de julio de 1493 escribió una carta al rey Juan II de Portugal por encargo de Maximiliano II, quien invitaba al monarca a buscar Catay. Es posible que la teoría expuesta en dicha carta influyera en la concepción de Colón sobre su propio descubrimiento. Por otra parte, Münzer contempló en Sevilla a los indios traidos de América y conversó en Madrid con el P. Bernardo Boil, acompañante de Colón en su segundo viaje. Lo escrito por Münzer constituye uno de los primeros testimonios sobre el descubrimiento de América.

J. Münzer es el autor de uno de los relatos de viajes más importantes por la Península Ibérica. Realizó el viaje en los años 1494-1495, pero su relación manuscrita quedó inédita hasta el año 1924; en efecto, en esta fecha el hispanista Ludwig Pfandl (1) daba a las prensas el viejo manuscrito en su lengua original, el latín, acompañado de una introducción y de escasas, pero interesantes notas. Muy poco después el histo-

(1) Jerónimo MÜNZER, "Itinerarium Hispanicum 1494-1495», Revue Hispanique, Ed. Del texto latino y notas a cargo de L. Pfandl, XLVIII, 1920, págs. 1-179. 
riador J. Puyol (2) ofrecía una primera traducción al castellano, bastante defectuosa, por cierto. El interés del relato hizo que en 1951 el latinista J. López del Toro (3) intentara una nueva versión, que mejoró mucho la de Puyol.

Antes de entrar en el tema concreto que nos ocupa es conveniente referirnos a la vida y obra de nuestro autor, dado que en las introducciones de las traducciones de Puyol y López de Toro apenas son tratadas.

\section{Datos biográficos}

En 1920 L. Pfandl escribía las siguientes palabras: «Con las noticias sobre la vida de Jerónimo Münzer ocurre como con muchos autores de la antigüedad de Grecia y Roma: lo poco que sabemos de su origen, juventud, formación, actividad profesional, sucesos de su edad madura y finalmente de su muerte, nos ha llegado en forma de alusiones y noticias escasas y dispersas por aquí y por allá» (4).

A pesar de la erudición y de la diligencia del gran investigador, no habían llegado a su conocimiento interesantes descubrimientos sobre la vida de Münzer llevados a cabo por W. Lochner (5) y el Dr. Fromman (6); este último descubrió una autobiografía de Münzer escrita en 1507, un año antes de morir en la que se contienen importantes datos de su vida; en $1923 \mathrm{~J}$. Fischer (7) publicó en la revista española Razón y Fe un interesante artículo en el que utilizaba estos datos.

Un nuevo gran descubrimiento estaba reservado a E. P.

(2) Jerónimo MüNZER, “Viaje por España y Portugal en los años 1494-1495", versión del latín por J. Puyol, Boletín de la Real Academia de la Historia, LXXXIV, 1924, págs. 32-119 y 197-279.

(3) Jerónimo MünZER, Viaje por España y Portugal, 1494-1495, Trad. de J. López de Toro y Prólogo de M. Gómez Moreno, Madrid, 1951.

(4) Ludwig PfandL, [1], pág. 145

(5) W. Lochner, «Die Münzer, Hieronymus Holzschule und die Funtenbache", Anzeiger für Kunde der deutschen Vorzeit, n. ${ }^{\circ}$ 97-106, págs. 1875-1880.

(6) Fromman, "Dr. H. Monetarius», Anzeiger für Kunde der Deutschen Vorzeit, n. ${ }^{\circ}$ 357-359, pág. 2879.

(7) J. FISCHER, «El Dr. Jerónimo Münzer (Monetariu 1508). Su viaje por España. 1494-1495", Razón y Fe, LXVI, 1923, págs. 81-101.

R. I., 1996, n.o 207 
Goldschmidt (8), quien estaba llevando a cabo la historia de la biblioteca del castillo de Nikolsburg; en efecto, Goldschmidt pudo comprobar que una buena parte de dicha biblioteca procedía de la de J. Münzer; concretamente identificó 7 manuscritos y 165 obras encuadernadas en 100 volúmenes por el mismo encuadernador que trabajó para $\mathrm{H}$. Schedel; el hallazgo no termina ahí, ya que en los libros se encuentran valiosas anotaciones de Münzer, que ofrecen detalles sobre el lugar y la fecha exacta de la adquisición, así como sobre aspectos del contenido. Con todo este material pudo Goldschmidt trazar una magnífica biografía de Münzer, que publicó en 1938.

En contra de Pfandl (9), que había establecido el año 1460 para el nacimiento de Münzer, Godschmidt (10) rebajó notablemente dicha fecha hasta el año 1437; en lo que si hay unanimidad es en el lugar de nacimiento, Feldkirch, ya que así consta tanto en el título dado por Schedel al relato del viajero, como en el epitafio de su tumba en el cementerio de S. Sebaldo de Nuremberg. Pertenecía a una respetada pero humilde familia, por lo que sólo pudo realizar sus estudios gracias a la ayuda de alguna persona piadosa; en efecto, en 1464 aparece matriculado en Leipzig y en 1466 recibe el título de bachiller; en 1470 es Magister artium y pasa a ser profesor en la misma Universidad de Leipzig.

La formación de Münzer en esta Universidad se inserta dentro del movimiento humanista, pues en aquellas fechas era allí profesor el italiano Jacobus Publicius, cuyas explicaciones de Terencio debieron ejercer una influencia decisiva en el ambiente universitario del momento.

También en Leipzig empezó Münzer sus estudios de medicina, pero la meta soñada por los futuros médicos era entonces Italia, ya que allí estaban permitidas las disecciones de cadáveres; hacia la Universidad de Pavía se encaminó el año 1476, tras haber desempeñado la docencia durante dos años en su ciudad natal; en Pavía obtuvo el doctorado en medicina en 1478, y ese

(8) E. P. GoldschmidT, Hieronymus Münzed und seine Bibliothek, Londres, The Warburg Institute, 1938.

(9) Pfand, [4], pág. 146.

(10) GoLDSCHMIDT, [8], pág. 13. 
mismo año fue contratado como médico en Nuremberg, según consta en un documento del Consejo de dicha ciudad.

Una vez asentado en Nuremberg, pronto contrajo matrimonio con Dorotea Kieffhaber, de familia acomodada, y el ejercicio de su profesión le proporcionó reputación y buenos ingresos; pero esta situación duró poco, ya que en el año 1483 se desencadenó en la ciudad una terrible peste; a instancias del Consejo los médicos de la población redactaron un escrito en el que exponían medidas higiénicas y profilácticas para combatir la enfermedad; lo primero que aconsejaban era huir de la ciudad en busca de aires más saludables; y esto es precisamente lo que hizo el Dr. Münzer, quien el 12 de septiembre de 1483 salió de Nuremberg hacia Italia; la vuelta tuvo lugar el 24 de enero de 1484.

La situación de 1483 se volvió a repetir en 1494, según palabras del propio Münzer:

Después en el año 1494, al iniciarse una nueva peste, queriendo buscar de nuevo el antiguo remedio de la huida y dando vueltas al asunto, elegí compañeros de viaje a unos jóvenes de buena familia, hijos de ricos comerciantes, que dominaban el italiano y el francés: Antonio Herwart de Augsburgo, Caspar Fischer y Nicolás Wolkenstain.

Esta vez, la meta del viaje, fue la Península Ibérica, en la que se habían producido acontecimientos trascendentales: hacía poco que había sido reconquistado el último baluarte de los musulmanes, el reino de Granada, y hacía poco también que habían llegado a España hombres de tierras desconocidas; además el rey de Portugal, Juan II, era primo de Maximiliano de Austria, quien estaba muy interesado en los descubrimientos de los portugueses.

El viaje se inició el 2 de agosto de 1494, y a grandes rasgos el recorrido del viaje fue el siguiente: varias ciudades alemanas, suizas y francesas; ya en la Península: Cataluña, Valencia, Murcia, Andalucía, Portugal, Galicia, Extremadura, Castilla, Aragón y Navarra; la llegada a Nuremberg tuvo lugar a finales de abril de 1495, tras haber visitado Francia por segunda vez y Bélgica.

No se sabe mucho sobre los últimos años de la vida de Münzer, si bien se conocen algunos hechos importantes, como 
el matrimonio de su hija Dorotea con el Dr. J. Holzschuher, a quien Münzer apreció siempre; él fue precisamente el heredero de la mayor parte de su valiosísima biblioteca; Dorotea aportó a su matrimonio una elevadísima dote, lo que demuestra el éxito alcanzado por el Dr. Münzer en su trabajo. Hacia finales de septiembre de 1505, y tras veintiún años de matrimonio Münzer perdió a su esposa. En los comienzos del año siguiente en compañía de su hija y de su yerno visitó su ciudad natal, a la que hizo donación de una parte considerable de sus libros. El 27 de agosto de 1508 tuvo lugar la muerte de Münzer, siendo enterrado en el cementerio de S. Sebaldo en compañía de su esposa.

Gracias a Schedel conocemos el epitafio redactado en latín y recogido por Goldschmidt (11): «Sepultura dedicada a la memoria y a la piedad de Jerónimo Münzer, hombre muy bueno, ilustrísimo doctor en filosofía y medicina. Fue un excelente protector de sus amigos y tan servicial para todos los hombres, que no sólo ayudó con su consejo y sabiduría a los más ricos, sino que también espontáneamente dio dinero a los necesitados de forma gratuita. Recorrió casi toda Europa para ver las ciudades y las variadas costumbres de sus habitantes».

\section{El Círculo Humanistico de Münzer}

Para Pfandl (12), Münzer no fue un humanista; sin embargo, el argumento aducido creo que no tiene mucha fuerza; en efecto, afima Pfandl que en su viaje por España no ofrece muchos datos sobre bibliotecas, impresores, manuscritos de autores griegos y romanos, etc. A esto se puede contestar que Münzer era un hombre de múltiples intereses: era médico, naturalista, geógrafo, astrónomo, historiador, etc.; casi podemos decir que era el típico homo universalis renacentista; también se puede afirmar que el objetivo de su viaje no era el descubrir nuevos manuscritos de autores clásicos; en consecuencia, al tener que atender a tantas solicitudes, no es extraño que dejara de anotar

(11) Ibidem, pág. 104.

(12) PfandL, [4], pág. 150. 
ciertos datos que a Pfandl le parecían de especial importancia; con todo, ofrece algunos detalles, v. gr. en el Monasterio de Guadalupe y en la Catedral de Toledo; además, supo apreciar el creciente interés hispano por esos estudios, como ponen de manifiesto sus mismas palabras:

Ahora, por el contrario, empieza a tener fuerza la elocuencia, sobre todo, entre próceres y nobles de España; estimulados por su ejemplo, todos los ciudadanos y los clérigos se dedican a estos estudios humanísticos... los estudios humanísticos se despiertan en toda España.

Completamente contraria a la de Pfandl es la postura de Goldschmidt (13), para quien Münzer es el típico representante del temprano humanismo de Nuremberg. Esta tesis me parece evidente, pues basta examinar la composición de su biblioteca para darse cuenta de que en ella resplandecen los ideales renacentistas.

También es muy ilustrativo a este respecto tener en cuenta quiénes fueron los amigos de Münzer, entre los que hay que destacar al poeta Conrad Celtes y a los hermanos Hermann y Hartmann Schedel. Con Celtes, el poeta neoplatónico, mantuvo Münzer trato epistolar y una gran amistad, ya que seguramente fue él quien mejor comprendió su poesía y sus ideas filosóficas.

En cuanto a los hermanos Schedel, Hermann había logrado aglutinar en Augsburgo un círculo de personas interesadas en los ideales renacentistas; lo mismo intentó hacer durante su estancia en Nuremberg, en donde contó con el apoyo de Münzer y de su hermano Hartmann; la biblioteca de éste fue el lugar de reunión de todo el círculo humanístico de dicha ciudad; Hartmann Schedel ha pasado a la historia por su famoso Liber Chronicarum, impreso en Nuremberg en 1493, y adornado con 2.000 grabados de Miguel Wohlgemuth, el maestro de Durero, y de Guillermo Pleydenwurff; precisamente en la realización de este libro tomó parte Münzer, según veremos en el siguiente apartado.

(13) GoLSDChMIDT, [8], pág. 35. 


\section{MÜNZER y LA GEOGRAFía}

Ya hemos aludido anteriormente a la amplitud de la formación científica de Münzer, en la que, además del dominio de la medicina, entraban conocimientos de astronomía, geografía, ciencias naturales, etc. Ahora vamos a tratar de su actividad en el campo de la geografía, ciencia en la que destacaron los eruditos de la ciudad de Nuremberg. En ella desarrolló parte de su labor científica el gran astrónomo, matemático y geógrafo llamado Joannes Muller, Regiomontanus. Gracias a su impulso nacieron dos importantes realizaciones, en las que colaboró Münzer: el globo terráqueo de Martín Behaim y el primer mapa de Alemania.

M. Behaim fue un marino amante de la aventura, de quien no sabemos mucho; perteneció al círculo de Schedel y Münzer; se casó con la hija de un flamenco; fijó su residencia en Portugal, en donde el rey Juan II le hizo miembro de la Junta de Mathemáticos; en 1484 tomó parte en un viaje a Guinea y a su vuelta fue admitido por el rey en la Orden de Cristo; realizó una misión en Flandes por encargo de Juan II, en la que al parecer no tuvo éxito. En la casa de sus suegros se hospedó Münzer durante su estancia en Lisboa, como él mismo anota en su viaje:

Teníamos el hospedaje en una grandísima y magnífica casa del rey, en las habitaciones del suegro de Martín Behaim, Don Jadoco de Murder, de Brujas, hombre noble y gobernador de las islas Fayal y Pico... Fuimos espléndidamente tratados.

En el año 1490 volvió Behaim a Nuremberg, y en los años siguientes fue cuando debió trabajar en la realización del globo, en el que junto a datos procedentes de portularios se perciben informaciones de geógrafos antiguos como Ptolomeo, Estrabón, etc. A propósito de este hecho los investigadores alemanes trataron de descubrir los colaboradores de Behaim, que no podían ser otros que Schedel y Münzer; Sauber (14) localizó en la biblioteca de Schedel un manuscrito titulado De

(14) R. STAuber, "Die Schedelsche Bibliothek», Studien und Darstellungen aus der Gebiete der Geschichte, n. 2 y 3, 1908, pág. 60. 
globo spherico terre, en el que se trata del origen del globo de Behaim y se incluyen trozos de la obra Asia de Eneas Silvio Piccolomini, el Papa Pío II; basándose en esto, atribuye a Schedel la colaboración de Behaim en la realización del globo; pero otro investigador, Granert (15), va más allá; partiendo del hecho de que Schedel solicitó el consejo y la colaboración de Münzer para la parte geográfica del Liber Chronicarum, llega a atribuir la paternidad del manuscrito De globo spherico terre a Münzer; Schedel sería como en otros casos, mero copista; esta tesis es compartida por Fischer (16).

La segunda empresa geográfica de Münzer tiene relación con la gran obra histórica preparada por Schedel, el Liber Chronicarum, cuya edición latina apareció el 12 de julio de 1493; se trata de una compilación de fuentes antiguas, en la que al final inserta una moderna descripción de Europa debida a Eneas Silvio Piccolomini; en esta última parte colaboró Münzer haciendo correcciones y adiciones; entre éstas, la más importante es la inclusión de la noticia de la conquista de Granada por los Reyes Católicos, de la que tratará ampliamente en el relato del viaje. Pero no termina ahí la colaboración de Münzer en la magna obra de Schedel; en efecto, él fue el autor del mapa de Alemania incluido en los folios 299-300 del Liber Chronicarum; se trata del primer mapa de Alemania ampliamente divulgado por la imprenta, ya que antes existía uno manuscrito atribuido al Cardenal Nicolás de Cusa, y otro, grabado en 1492, del se conservan unos seis ejemplares y que ha. suscitado grandes controversias, que no es preciso examinar aquí.

La importancia de Münzer como geógrafo puede contrastarse en estas autorizadas palabras de Goldschmidt (17): «Por lo demás, de lo dicho se puede conjeturar que Münzer tuvo en Alemania como cartógrafo cierto peso".

Como ilustración y prueba de la participación de Münzer en la obra de Schedel quisiera ofrecer unos párrafos de una

(15) H. GRANERT, «Die Entdeckung eines Verstorbenen Zur Geschichte der grossen Landerentdeckungen", Historisches Jahrbuch der Görresgesellschaft, n. ${ }^{\circ} 29,1908$, págs. 304-337.

(16) FischeR, "Der Nürnberger Arzt Dr. H. M. Aus Feldkirch als Mensch und Gelehrter», Stimmen der Zeit, n.o 96, 1919, págs. 149-168.

(17) GolsdchmidT, [8], pág. 58.

R. I., 1996, n.o 207 
larga carta escrita en latín por Münzer a Schedel, conservada en el ejemplar manuscrito del Liber Chronicarum; a mi parecer, dicha carta debió ser pensada como prólogo a dicha obra, aunque por razones desconocidas no llegó a publicarse como tal. La traducción la hago sobre el original publicado por Goldschidt (18).

Has insertado también en tu obra, mi querido Hartmann, como muy apropiada a esta publicación, la historia que el Papa Pío escribió hace tiempo sobre Europa y las empresas llevadas a cabo en ella. Como la encontraste hecha pedazos, mutilada y tan alterada en muchos pasajes que el propio Pío se horrorizaría si la leyera, quisiste que yo en vista de tu aprecio la revisase y la corrigiese. A pesar de la dificultad, sin embargo, para ayudar a los defensores de ia lengua latina y para que escuches a Pío hablando en sus eruditísimas palabras, he trabajado con todas mis fuerzas y me he esforzado con cuidado en corregir los muchos errores advertidos, debido quizá a la negligencia de los copistas o al desconocimiento de los correctores. Ahora me parece haberlo conseguido si lo lees con atención.

De modo parecido he añadido al final un mapa general de los lugares de Alemania con sus límites orientales y occidentales, a fin de que, puestas ante los ojos, quedasen más claras la longitud y latitud de Alemania.

\section{Análisis del viaje por España y Portugal}

Al trazar la biografía de Münzer hemos citado sus propias palabras para conocer el motivo de su viaje a la Península Ibérica: de nuevo la peste había sido la causa de su salida de Nuremberg. Sin embargo, para Pfandl (19), el motivo más importante debió ser otro: hablar personalmente con el rey de Portugal y llevar a Maximiliano una respuesta a la carta que el propio Münzer escribió a Juan II; tal carta, fechada el 14 de julio de 1493, seguramente fue llevada al rey portugués por M. Behaim; en ella, en nombre de Maximiliano, invita a

(18) Ibidem, pág. 109.

(19) PfandL, [4], págs. 147-149. 
Juan II a navegar por el mar occidental y así llegar al mundo oriental, concretamente a Catay (China), que debía estar a pocos días de navegación. De esta carta se conservan las siguientes copias: $1 .^{a}$ El texto latino original del comienzo de la carta es una hoja escrita por Schedel; 2. ${ }^{\text {a }}$ Traducción completa al portugués, realizada por el dominico Álvaro de Torre y publicada en su libro Tractado la spera do mundo; 3. ${ }^{\text {a Traducción al }}$ portugués con variantes ortográficas respecto a la anterior, publicada en la obra Regimento do estrolabio e do quadrante pera saber la declinaçam e ho logar do sall em cada huum dia $e$ asy pera saber ha estrella do norte; 4. ${ }^{\text {a }}$ Traducción al portugués distinta de las dos anteriores, encontrada por el P. SilvaTaranca en la biblioteca Rossiana.

En el apéndice ofrecemos una traducción castellana de la carta de Münzer, realizada sobre el original latino en la parte conservada y en el resto sobre la traducción portuguesa publicada en Regimento do estrolabio.

Como prueba de su hipótesis, Pfandl presenta algunos pasajes del propio Münzer.

[el rey] Me invitó cuatro veces a su mesa y me hablaba de variados asuntos, mostrándose muy amable... Al despedirnos de él, el día de Sta. Catalina, después de la cena en el palacio me estrechó en sus brazos.

Cuatro veces me llamó a su mesa y, mientras los demás estaban en silencio, me hablaba durante ocho horas de Cosmografía en la que está impuesto, de Medicina y de otros temas.

De nuevo nos pusimos en camino desde Sevilla siguiendo el Guadalquivir, y llegamos al palacio del rey de Portugal, donde fuimos informados por su majestad real acerca de los asuntos de Etiopía y de las tierras del sur (20).

A pesar de este buen recibimiento y del trato afectuoso del rey, según Vignaud (21), su contestación a la propuesta de Maximiliano habría sido negativa, y ésta sería la razón por la que Münzer guardó silencio sobre el tema en el relato del viaje.

(20) Ibidem, págs. 148-149.

(21) M. Vignaud, Histoire critique de la grande enterprise de Chistophe Colomb, 2 vols., Paris, H. Welter, 1911, vol. II, pág. 464.

R. I., 1996, n. 207 
Por su parte, Pfandl (22) cree que Münzer o bien contestó de palabra a Maximiliano o bien presentó un informe separado, que por alguna razón no copió Schedel.

A la tesis central de Pfandl, esto es, que la causa principal del viaje de Münzer fue llevar la contestación de Juan II a Maximiliano, creo que pueden ponerse algunas objeciones: $1{ }^{a} \mathrm{Si}$ M. Behaim fue quien llevó la famosa carta al rey de Portugal, lo más lógico sería pensar que el propio Behaim, amigo del rey, llevase la respuesta a Maximiliano. 2. ${ }^{\text {a }} \mathrm{Si}$ Münzer fue enviado con la misión de llevar la respuesta a Maximiliano, creo que el itinerario del viaje debía haber sido más directo, tanto en la ida como en la vuelta, al menos si tenían alguna prisa en conocer la contestación; pues bien, la salida de Nuremberg tuvo lugar el 2 de agosto de 1494 y la entrada en España el 17 de septiembre; demasiado tiempo para un viaje de cierta urgencia. Después de la entrevista con Juan II, en vez de emprender un camino directo hacia Alemania, irán a Santiago, luego bajarán a Guadalupe y desde allí irán a Toledo, Madrid, Zaragoza, Pamplona, Francia y Bélgica. 3.a De las propias palabras de Münzer parece deducirse que tenía más interés en entrevistarse con los Reyes Católicos que con Juan II. Si damos fe a sus palabras el objetivo del viaje sería conocer sobre el terreno los grandes hechos llevados a cabo por los monarcas hispanos y, sobre todo, la conquista de Granada, a la que Münzer dedicará los más amplios comentarios de su relato. Las palabras a las que aludía se encuentra en el discurso pronunciado ante los reyes en Madrid.

Sacratísimos y potentísimos reyes: la grandeza de las hazañas realizadas por vuestras majestades es conocida por todo el universo, y ha conseguido que los príncipes y demás nobles alemanes se pregunten con gran admiración de qué forma los reinos de España, que en los tiempos pasados parecían casi desmoronados, destruidos y quebrantados por causa de los levantamientos intestinos, los odios ocultos y el interés privado, con una suerte repentina y en corto espacio de tiempo han pasado de la más grande discordia a una paz y tranquilidad tan grandes y a una óptima situación política. Muchos no creyeron estos hechos. Por eso y con el favor de

(22) PfandL, [4], pág. 149. 
nuestro serenísimo rey Maximiliano y de los demás nobles de Alemania, aceptando con mi comitiva esta misión, salí desde las más alejadas tierras de Alemania y entré en España, para poder ver con mis propios ojos lo que había oído.

Por todo ello creo que el viaje de Münzer a la Península estuvo motivado por diversas razones, que podemos sintetizar así: 1. ${ }^{a}$ Estallido de la peste en Nuremberg; 2. ${ }^{a}$ Deseo de conocer en detalle y profundidad la conquista de Granada, sobre la que ya había escrito Münzer para el Liber Chronicarum; 3. ${ }^{a}$ Deseo de conocer a los Reyes Católicos, por quienes el viajero sentía una

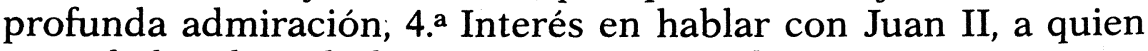
antes había dirigido la carta ya mencionada.

A todas estas motivaciones puede añadirse como causa desencadenante el espíritu aventurero y deseoso de conocer nuevas tierras propio de Münzer.

\section{El descubrimiento de América}

No puede caber ninguna duda de que Münzer tuvo una información privilegiada sobre el descubrimento de América o, mejor dicho, de las islas índicas, según sus propias palabras; en efecto, en Madrid permanecio ocho días, durante los cuales tuvo largas conversaciones con el P. Bernardo Boil, que había acompañado a Colón en su viaje; así lo recuerda Münzer:

Tiene [Madrid] también tres monasterios: de menores, de predicadores, y una nueva fundación de la orden de los menores, al frente de la cual está el hermano Bernardo Boil, aragonés, que fue enviado a las islas índicas como verdadero explorador. Tuve un trato muy familiar con él en Madrid y me acompañó ante el rey. Me habló de las islas.

Así mismo tuvo ocasión de ver en Sevilla a los indios enviados por Colón en febrero de 1494 en doce naves a las órdenes de Antonio Torres; así lo cuenta:

...y, volviendo por el altísimo monte de Portalón, llegamos a Sevilla, donde tenía lugar un nuevo milagro. Vimos unos hombres nuevos, desconocidos en nuestro mundo, que ha- 
bían sido llevados allí desde las islas índicas, descubiertas bajo vuestros auspicios. iHecho increíble y desconocido por muchos!.

En Évora fue invitado cuatro veces a la mesa del rey Juan II, que a su vez había recibido también a Colón al término de su primer viaje. iQué conversaciones tan interesantes tendrían lugar entre dos personas tan bien informadas sobre los descubrimientos de nuevas tierras! A pesar de estos hechos, apenas dice nada Münzer en su relato acerca de un tema tan querido para él. Ello se debe a que el viajero redactó un escrito especial, que desgraciadamente se ha perdido; en efecto, en el viaje de vuelta a su paso por Francia cuenta lo siguiente:

De él [Bernardo Boil] hablé en el Tratado sobre el descubrimiento de las Indias.

No termina aquí la relación de Münzer con el descubrimiento, ya que él tomó parte en la realización de globo de M. Behaim y, sobre todo, él fue quien escribió la famosa carta a Juan II; esto demuestra que Münzer tuvo una participación importante en el proyecto del paso a Oriente por el mar occidental atribuido a $\mathbf{M}$. Behaim; de otra forma, ćcómo se explica que fuera él el redactor de la carta, cuando la podía haber escrito M. Behaim perfectamente en portugués? Y esto sería lo normal, dado que Behaim había tenido muy buenas relaciones con Juan II y él mismo iba a ser quien llevara la carta al monarca.

Con ello entramos en el apasionante tema de las relaciones entre Behaim-Münzer y Colón, cuestión intrincada, en cuyo punto de partida está la carta, que supuestamente escribió en 147,4 el médico y astrónomo florentino Paulo del Pozzo Toscanelli al canónigo de Lisboa Fernando Martins; en ella expone que es posible llegar a oriente navegando por occidente; para algunos investigadores, que defienden la autenticidad de dicha carta, Colón llegó a conocerla si bien de forma irregular según mantiene también Gil (23): "Porque, a decir verdad, parece

(23) Juan GIL y Consuelo VALERA, Cartas de particulares a Colón y relaciones coétaneas, Madrid, Alianza, 1984, pág. 132. 
que tiene toda la razón una serie de investigadores, entre los que se encuentra Ruge, Haebler, Goodrich y Madariaga, al sospechar que el genovés se hizo con esta información de manera poco santa".

En caso de ser auténtica la carta de Toscanelli, podría ser también la fuente de la carta escrita por Münzer al rey de Portugal, en la que expone ideas parecidas a las del italiano. Sobre esta carta fechada el 14 de julio de 1493 se puede hacer una pregunta importante, chabía llegado a Nuremberg la noticia del viaje y el regreso de Colón? La respuesta es afirmativa para Vignaud (24), quien pone como argumento principal las relaciones de la ciudad de Nuremberg con los comerciantes flamencos, de los que había gran número en las islas portuguesas.

Por mi parte, me inclino por una respuesta negativa para la que propongo los siguientes argumentos: 1. ${ }^{\circ}$ En el Liber Chronicarum, publicado el 13 de julio de 1493, no se hace mención ni de la salida ni del regreso de Colón; desde el regreso habían pasado sólo cuatro meses, pero tal vez suficientes para incluir una noticia de tanta importancia, sobre todo, para el círculo de Schedel, Behaim y Münzer; 2. Si en Nuremberg se hubiera sabido que, tras el desembarco en Lisboa, Colón había sido recibido por Juan II, resultaría poco lógico proponer a este monarca un viaje de descubrimiento ya llevado a cabo, y del que estaba perfectamente informado; 3. $\mathrm{Si}$ el emperador Maximiliano hubiera querido que Juan II compitiera con los Reyes Católicos en el descubrimiento realizado por Colón, tal vez Münzer en su carta debía haber hecho alusión a su viaje; 4. Sería poco ético para una persona de la categoría de Münzer proponer como nuevo algo ya conocido; $5 .^{\circ}$ En el discurso pronunciado por Münzer ante los Reyes Católicos en Madrid se encuentran estas palabras:

Vimos unos hombres nuevos, desconocidos en nuestro mundo, que habían sido llevados allí desde las islas índicas, descubiertas bajo vuestros auspicios. iHecho increíble y desconocido para muchos!.

¿No estaría aludiendo con ellas al retraso en la divulgación de la noticia del viaje colombino?

(24) Vignaud, [21], pág. 454. 
Una pregunta importante se puede plantear con relación a la carta escrita por Münzer y a la invitación de Maximiliano en ella contenida: ¿’Por qué no accedió Juan II a realizar un viaje parecido al de Colón, sobre todo, teniendo en cuenta que tenía información directa del propio descubridor? En efecto, Colón desembarcaba en Lisboa y cinco días después, esto es, el 9 de marzo de 1493 era recibido por el rey.

Tal vez la respuesta haya que buscarla en la deteriorada salud del monarca, como se comprueba por las palabras de Münzer:

Tenía mal color, ya que después de la muerte de su hijo Alfonso por una caída de caballo siempre estuvo mal. Hay que temer que sea hidropesía, pero que no lo sea.

De hecho, el rey portugués moría unos meses después, el 25 de octubre de 1495.

Téngase presente que unos años antes, cuando Colón le presentó su proyecto, a pesar de que la comisión nombrada al efecto se mostró contraria al descubridor, envió en secreto una carabela que tuvo que volver debido al mal tiempo. Por otra parte, las relaciones entre Juan II y Colón continuaron siendo buenas, como lo demuestran la estancia de Colón en Lisboa en 1488 y la carta de amparo que le envió el rey el 20 de marzo del mismo año.

Por lo expuesto hasta ahora se puede apreciar que la autenticidad de la carta de Toscanelli es fundamental para juzgar la relación entre Behaim-Münzer y Colón. En caso de una solución positiva es fácil conjeturar que las ideas de los tres proceden del sabio florentino. Pero si la carta no es auténtica se abre una seriẹ de interrogantes difíciles de solucionar: ¿Qué es realmente lo que pretendía Colón? ¿De dónde deriva su proyecto? ¿Es anterior a la construcción del globo de Behaim y a la carta de Münzer? ¿Influyó Colón sobre Behaim-Münzer o viceversa? A todas estas preguntas trató de dar solución $\mathrm{H}$. Vignaud, quien defiende la no autenticidad de la carta de Toscanelli. Para Vignaud (25): "Colón dijo todo lo que Behaim y Münzer avanzaron, uno en su globo y el otro en su carta al rey Juan; pero él lo dijo

(25) Ibidem, págs. 475-478. 
después de ellos, o, al menos, si lo dijo antes, no tenemos ninguna prueba de hecho, mientras que poseemos numerosas indicaciones que tienden a mostrar que su tesis es posterior a sus descubrimientos. Si nos referimos sólo a los documentos, no puede haber duda, puesto que vemos por el globo de Nuremberg, construido en 1493, y por la carta Münzer, escrita en 1493, que desde esa época el proyecto de Behaim estaba completamente razonado, mientras que Colón expuso su tesis sólo en las cartas que no datan más que de 1498 y $1503 \ldots$ Si en el estado actual de la cuestión no se puede solucionar definitivamente afirmando que Colón debe a Behaim todo lo que dice de esencial sobre la posibilidad de pasar a Asia atravesando el Atlántico, hay al menos fundamento para sostener que, en tanto no se tenga la prueba de que Toscanelli escribió realmente la carta que se le atribuye, y de que la empresa de 1492 tenía las Indias por objetivo, esta suposición es la que mejor concuerda con los hechos auténticamente establecidos».

Para solucionar estos problemas Vignaud trató también de averiguar cuál fue el verdadero proyecto de Colón, que no sería otro que el descubrir nuevas islas; solamente cuando llegó a las Antillas, a una distancia mucho mayor de la prevista creyó que estaba en la India y en la proximidad de Catay; a su vuelta declaró que venía de la India y se dedicó a buscar las razones teóricas de su creencia; después de su tercer viaje completó su teoría de la pequeñez del globo y de la proximidad de las Indias, formulada por primera vez en 1498.

Por mi parte, el razonamiento crítico de $\mathrm{H}$. Vignaud me parece bastante convincente, y solamente podría añadir que el testimonio de Münzer confirma que Colón a la vuelta de su primer viaje dijo que venía de las islas índicas, ya que éstas son las palabras empleadas por Münzer, quien, a su vez, las oiría de testigos muy cualificados como el P. Boil y el rey de Portugal.

Como resumen y precapitulación podemos afirmar que es muy posible que la teoría formulada por Münzer en su carta influyera en la concepción colombina, tal como aparece a partir de 1498; por esta razón y las aducidas al principio de este apartado Münzer debe ocupar una posición importante en lo relativo al descubrimiento. 


\section{APÉNDICE}

\section{CARTA del Dr. J. Münzer al REy JuAN II. 1493}

Al serenísimo e invictísimo Juan, rey de Portugal, de los Algarves, de la Mauritania Costera, y primer descubridor de las islas de Madera, las Afortunadas, las Casitérides y las Azores, humildemente se encomienda Jerónimo Münzer, doctor en medicina, alemán.

Porque hasta este momento has aceptado de tu tío, el serenísimo infante Enrique, la proclama de descubrir el globo terrestre, no has escatimado ningún esfuerzo ni ningún gasto, $\mathrm{y}$ con tu diligencia te has hecho tributarios el mar de Etiopía y de Guinea, así como los pueblos costeros hasta el trópico de Capricornio con sus mercancías, como el oro, los granos del Paraíso, la pimienta, los esclavos y otras. Con ese talento te has ganado alabanza, gloria inmortal y además enormes beneficios, y no hay duda de que en poco tiempo los etíopes, que bajo figura humana son como bestias al estar privados del culto divino, gracias a tu diligencia dejarán su bestialidad y llegarán a la práctica religiosa católica. Considerando estos hechos, Maximiliano, rey invictísimo de romanos, portugués de madre, ha querido por medio de mi carta, aunque tosca, invitar a tu majestad a buscar el riquísimo país oriental de Catay. Afirman, en efecto, Aristóteles al final del segundo libro Sobre el cielo, Séneca en el quinto de las Cuestiones naturales, Pedro d'Aully, el más docto de su tiempo, y un gran número de otros ilustrísimos hombres, afirman, digo, que el comienzo del oriente habitable está bastante próximo al fin del occidente habitable. Una prueba son los elefantes, que abundan en ambas regiones. De igual forma, las cañas, que la tempestad arroja desde la parte oriental hasta el litoral de las islas Azores. Hay además innumerables, por hablar así, y muy ciertos argumentos por los que como por demostración se conjetura que aquel mar puede ser surcado en pocos días en dirección al Catay oriental. Que no te perturbe Alfagrano y otros sin sexperiencia, que dijeron que sólo una cuarta parte de la tierra está por encima del mar, mientras que las otras tres están sumergidas en él. En efecto, en las cosas que se refieren a la habitabilidad de la tierra hay que fiarse más de la experiencia y de los relatos probables que de las imaginaciones fantásticas. Muchos autorizados astrónomos han negado que haya alguna tierra habitable bajo los trópicos y las regiones equinocciales. Con tu experiencia has probado que tales cosas son vanas y falsas. No hay ninguna duda de que la tierra no se extiende bajo el mar, sino por el contrario el mar está debajo. Se añade la redondez orbicular de la misma. Tienes también abundancia de recursos y riquezas, y tienes marinos muy sabios, que 
así mismo desean ganar inmortalidad y gloria. iCuánta gloria alcanzarás si haces el oriente habitable conocido a tu occidente! Y también cuánto beneficio te dará el comercio, pues harás las islas de oriente tributarias y muchas veces los reyes maravillados se someterán sin mucho esfuerzo a tu señorío. Ya te alaban como gran príncipe los alemanes, italianos, rutenos, polacos, escitas y los que habitan debajo de la estrella árida del polo ártico, como el gran duque de Moscovia; no hace muchos años que debajo de la sequedad de dicha estrella fue nuevamente descubierta la gran isla de Groenlandia, que tiene de costa 300 leguas y en la que habita muchísima gente del señorío del citado señor duque. Pero si llevas a cabo esta expedición, te elevarán en alabanzas como a un dios y otro Hércules; tendrás también, si te place, como compañero para este camino al señor Martín Behaim, especialmente designado por nuestro rey Maximiliano para realizar esto, y a otros muchísimos marinos experimentados, que desde las islas Azores surcarán el ancho mar con su esfuerzo, valiéndose del cuadrante, del cilindro, del astrolabio y de otros instrumentos; no les arredrará ni el frío ni la calma, y navegarán hacia el litoral oriental bajo una temperatura muy templada del aire y del mar; son innumerables los motivos por los que tu majestad puede ser estimada. Pero ¿de qué sirve espolear a quien corre? Y tú mismo eres de tal forma que examinas a fondo todas las cosas con tu diligencia y, por tanto, escribir mucho sobre este tema es impedir a quien corre que llegue al final. Que el Todopoderoso te mantenga en tu propósito y que, acabado el camino del mar por parte de tus caballeros, seas celebrado con la inmoralidad. Salud; Nuremberg, villa de la alta Alemania, a 14 de julio del año de la salvación 1493.

J. Münzer made a trip through the Iberian Peninsula during 1494 and 1495, about which he wrote an interesting account. His relation with American is based in several facts: Maximilian II had set him the task of writing a letter to the King John II of Portugal, which he did on July the $14^{\text {th }}$ of 1493 . It is possible that the theory expressed in that letter had influence in Colon's conception about his own discovery. On the other hand, Münzer gazed at the Indians of America in Sevilla and had a conversation with Bernardo Boil, Colon's companion in his second trip, in Madrid. What Münzer wrote is one of the early testimonies about the discovery of America. 